\title{
High power, low-noise, and multiply resonant photodetector for interferometric gravitational wave detectors
}

\author{
H. Grote ${ }^{a)}$ \\ Max-Planck-Institut für Gravitationsphysik (Albert-Einstein-Institut) und Universität Hannover, \\ Callinstrasse 38, 30167 Hannover, Germany
}

(Received 29 November 2006; accepted 18 March 2007; published online 8 May 2007)

\begin{abstract}
We have developed a new photodetector circuit for use in interferometric gravitational wave detectors. The circuit can detect high laser power with low noise and provide multiple outputs for different signal frequencies. The dynamic range of this circuit is increased in comparison with the photodetector design used until the end of 2005. (C) 2007 American Institute of Physics.
\end{abstract}

[DOI: 10.1063/1.2735559]

\section{INTRODUCTION}

Laser-interferometric gravitational wave detectors impose unique requirements on the detection of light. In the current generation of these interferometers, the most demanding application of a photodetector is the measurement of the light present at the output port (also called dark port) of the instrument. All of these interferometers employ a heterodyne readout technique, resulting in a light beam at the dark port carrying the desired displacement information in the amplitude of a beat signal. While the particular features of the light at the dark port depend on the individual parameters of an interferometer, typically there are requirements for a photodetector at this port: (a) Detect as high laser power as possible; (b) detect a possibly large signal content at a radio frequency, the detection frequency, with a noise floor dominated by the photon shot-noise of the detected light.

While high laser power is important to increase the shotnoise limited sensitivity of the interferometer, the detection of light has to be performed with a large dynamic range. In addition to these requirements, there can be constraints and further desirable features of the photodetector.

- Very large signals at radio frequencies other than the detection frequency may be present as well, and must not disturb the measurement at the detection frequency.

- For various purposes it might be desirable to have outputs of the photodetector optimized for radio frequencies other than the detection frequency.

- A sensitive light detection, preferably shot-noise limited, at audio frequencies and dc, is useful as well.

As Gray et al. pointed out in Ref. 1, there is no commercial photodetector able to provide the necessary combination of the above features. The main reason is that many commercial photodetectors are designed for optimal detection of low light powers. But even commercial detectors that can sense

\footnotetext{
${ }^{a)}$ Electronic mail: hartmut.grote@aei.mpg.de
}

more light are not optimal regarding the need to detect large signals at distinct radio frequencies with a very large dynamic range.

Consequently, all of the interferometric-detector groups currently running large interferometers have developed their own solution. All of the detectors employ light at a wavelength of $1064 \mathrm{~nm}$ and use indium-gallium-arsenide photodiodes with a high quantum efficiency. However, the electronic circuit designs around the photodiode differ in various aspects. While Sec. II gives a review of the approaches of the different groups, Sec. III introduces the new photodetector design, now used in the GEO600 interferometer. The measurements shown in Sec. IV indicate an increased dynamic range of the new circuit.

\section{OVERVIEW OF EXISTING CIRCUIT DESIGNS}

\section{A. Design principles}

Before having a look at discrete circuit designs, we want to illustrate the basic considerations about the important noise sources and related guidelines for the circuit design of a photodetector.

The most important quantity to optimize in the circuit design for a photodetector at the dark port of an interferometer is the dynamic range of the photodetector, which is simply speaking the maximal possible signal size, at a given frequency, divided by the noise of the detector (at the same frequency and in appropriate units). For example, a gravitational wave detector may yield light at the dark port, containing information equivalent to a displacement measurement of $10^{-19} \mathrm{~m}$ at $100 \mathrm{~Hz}$, in the presence of a rmsdisplacement signal of, e.g., $10^{-12} \mathrm{~m}$, which is already a required dynamic range of $10^{7}$, not accounting for necessary margin.

For the design of a photodetector, we have to consider the main noise sources, as well as the maximal signal size to be expected. In addition to the intrinsic photon shot noise, there are thermal noise and amplifier noise. In the normal run mode of a gravitational wave interferometer, we can assume a fixed light level at the dark port, exhibiting a fixed amount of shot noise. Taking into account any nonstationarity of the 


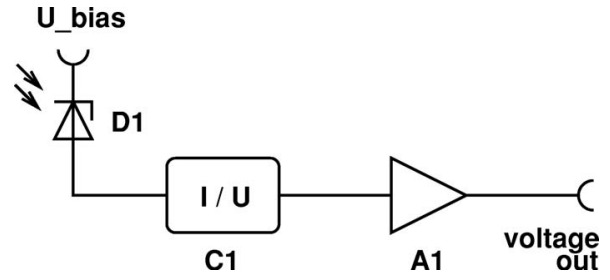

FIG. 1. Principle layout of a photodiode circuit. Unit C1 serves as a currentto-voltage converter. A1 is a voltage amplifier.

light power in the calculation of the effective noise level, ${ }^{2,3}$ we want the output of the photodetector to be limited by this noise, and no other. Thus we have to make sure that thermal noise and amplifier noise are sufficiently below the shot noise.

Naturally there is a limit to the maximal detectable photocurrent, given by properties of the photodiode, such as saturation and thermal overload. As far as these are specific for the type of photodiode used, they are not discussed here. For the purpose of circuit discussion, we assume a maximal light power to be detected by a photodiode that is either the total amount of light available or the maximal power that can be detected by a single photodiode.

In an approximate sense, a photodiode can be regarded as a current source, and typically some current-to-voltage converter has to be used, to generate a signal in the voltage domain, convenient for further processing.

Figure 1 shows a principle layout of a photodiode circuit consisting of a photodiode D1, a current-to-voltage converter $\mathrm{C} 1$, and a voltage amplifier A1. We can describe the conversion factor of $\mathrm{C} 1$ as $Z=U / I$ (which can be real or complex), and indeed a simple photodiode circuit would make use of a real resistor $R$ as current-to-voltage converter. The thermal (Johnson) noise of $\mathrm{C} 1$, which contributes to the total noise, is given by $U_{t}=\sqrt{4 k T R}$ with $k$ being the Boltzmann constant, $T$ the absolute temperature, and $R$ the real part of the impedance $Z$. The other relevant noise sources are the input-related voltage noise $U_{i}$ and current noise $I_{i}$ of amplifier A1. To estimate the contribution of $I_{i}$ to the overall voltage noise at the input to $\mathrm{A} 1$, the conversion of $I_{i}$ to a voltage has to be considered, which is a function of the converter $\mathrm{C} 1$, as well as of the implementation of A1.

The choice of the conversion factor $Z$ (or its real part $R$ ) is an important design consideration for this first stage of the photodiode circuit. Depending on the implementation of $\mathrm{C} 1$, $Z$ is a function of frequency, and, in particular, $Z$ has at least one local maximum for resonant photodetectors.

Figure 2 shows the principal contributions from the main noise sources mentioned above. Different noise voltages are shown as a function of the real part $R$ of the conversion impedance. The shot noise of the photocurrent can be regarded as a signal and thus has the same slope as any signal we want to detect, depending linearly on the value of $R$.

In the case of very low values of $R$, the output (of amplifier $A 1$ ) will be dominated by $U_{i}$. With increasing $R$, the signal (here the shot-noise voltage) rises linearly (note that the detected light level and thus the average photocurrent is nearly constant), while the thermal noise of the current-tovoltage converter rises only lineary with $\sqrt{R}$. Hence there is a

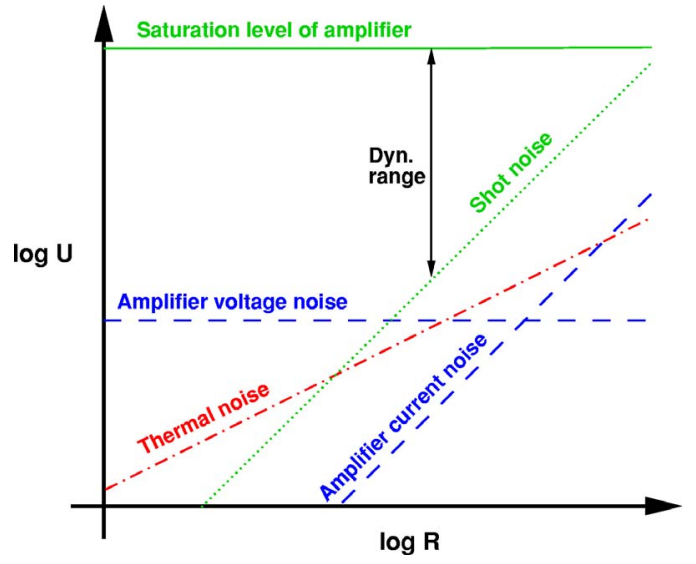

FIG. 2. (Color online) The main noise sources as functions of the conversion factor $R$, converting photocurrent to voltage.

minimum value of $R$ above which the shot noise dominates the other noise sources by at least a desired factor. However, $R$ should not be much larger than this value, because with increasing $R$, the maximal detectable signal size decreases, due to the unavoidable saturation level, which is located within A1 or in the circuitry beyond.

The slope of the amplifier current noise contribution in Fig. 2 is chosen for the case where C1 converts $I_{i}$ linearly with $R$ to a contributing noise voltage. Depending on the implementation of $\mathrm{C} 1$ this is not necessarily the case, but this linear behavior is a feature of the circuits discussed below.

In his publication on thermal noise, ${ }^{4}$ Johnson nicely states that the noise of thermal agitation is usually the predominant source of disturbance in a well constructed amplifier. In general, it is also true that thermal noise should be the predominant source of noise in a well constructed photodetector circuit; however, this is not necessarily so for the photodetector circuit we consider in our context. As mentioned above, the main difference is that often photodetectors have to be as sensitive as possible to small amounts of light, whereas in the case discussed here, we have a fixed (comparably large, which means several $10 \mathrm{~mW}$ or more) amount of light available for the photodetector.

An example should illustrate the optimization contained in the choice of $R$. Let us assume an amplifier A1 with $U_{i}$ $=2 \mathrm{nV} / \sqrt{\mathrm{Hz}}$, and a photocurrent of $20 \mathrm{~mA}$. If we want to dominate the amplifier noise $U_{i}$ with shot noise by a factor of 5 , we need a conversion resistance of $R \approx 124 \Omega$. This resistor will have a thermal noise of $U_{n} \approx 1.42 \mathrm{nV} / \sqrt{\mathrm{Hz}}$, (at room temperature) thus being below the amplifier voltage noise.

If we want to make the circuit to be limited by thermal noise, one option would be to use an amplifier with lower voltage noise. Typically this is only possible at the price of a larger gain of the amplifier. If we would use an amplifier with half the voltage noise and double gain, we would just be thermal noise limited, at the price of having reduced the dynamic range by a factor of 2 . Now we could reduce $R$ to restore our previous factor of the domination of noises by shot noise, and also restore the dynamic range. But as soon as we are limited by thermal noise, we cannot reduce $R$ by as much as the amplifier gain was increased. So in general there is nothing to be gained by reducing the amplifier voltage 


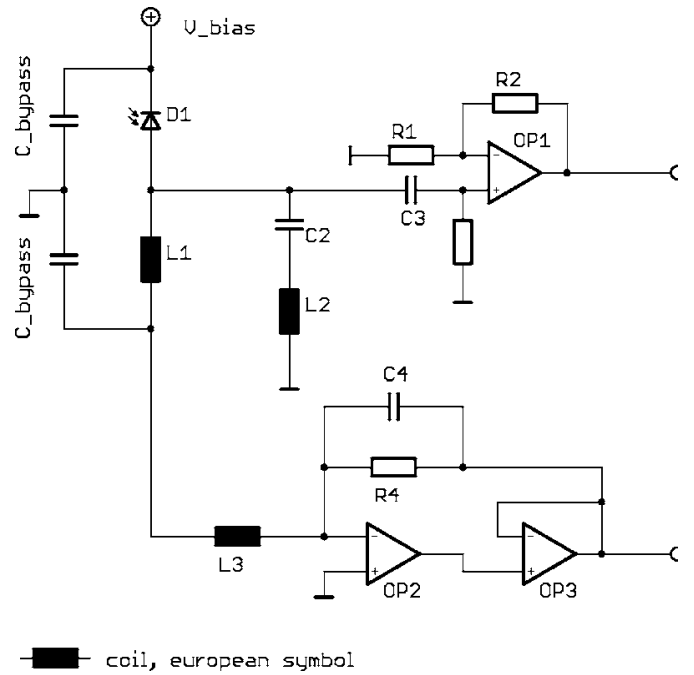

FIG. 3. The GEO600 dark-port photodetector design used until the end of 2005. The european symbol for coils is used as shown in the legend.

noise below a point where further reduction has to be paid with larger amplifier gain. Another option to make the circuit to be limited by thermal noise would be to increase $R$. However, in this case the dynamic range would be smaller.

In order to not further complicate the example, the role of amplifier current noise has been neglected here. As the current noise is intrinsic to the amplifier, it is clear that the choice of the amplifier is important for the optimization as well.

Of course there are other noise sources downstream of this first stage of signal processing, but these can best be accounted for by the appropriate amplifier gain of $A 1$. Naturally the gain of $A 1$ should not be higher than necessary to dominate the next noise sources downstream of this amplifier.

The photodiode circuits shown below focus on the relevant aspects of the discussion, with nonrelevant components being omitted for clarity. Accordingly, values of components are only given in case a particular feature of a circuit is to be emphasized.

\section{B. GEO 600}

Figure 3 shows the GEO 600 photodetector design used until the end of 2005, which we will refer to as the old GEO design. In this circuit the current-to-voltage converter discussed above consists of a resonant circuit around the photodiode, formed by the inherent capacitance of the photodiode (D1) and inductance L1. The signal voltage appears at the node between D1 and L1, and the resonant circuit is tuned to the detection frequency (15 MHz), by implementing $\mathrm{L} 1 \mathrm{as}$ a variable inductor. The photodiode is supplied with a reverse bias voltage in order to reduce the junction capacitance and increase the possible bandwidth of the diode. An additional notch filter consisting of a series resonant circuit formed by C2 and L2 is used to largely reduce signal voltages at twice the detection frequency, where a large signal is present. The signal is then high passed before being amplified by OP1.
The photocurrent for low frequencies (and dc, in particular) flows via L3 to the transimpedance amplifier stage formed by $\mathrm{OP} 2, \mathrm{OP} 3$, and R4. OP3 is a unity-gain buffer (BUF634), capable of driving sufficiently high currents.

A drawback of this circuit is that the signal voltage appears across the photodiode, which is disadvantegous for very large signal sizes. In this case the bias across the photodiode is modulated with the signal, which can lead to nonlinearities and shifts of the resonance frequency. Problems of this kind have been observed at GEO 600, which was the major reason to look for a different solution, which is described in Sec. III below.

There has been a radically different design of a photodetector for the dark port of GEO 600 as well, described in detail in Ref. 5. The photodetector therein consists of 16 photodiodes, in an arrangement where the rf photocurrents of all diodes are combined to yield a single rf output. While this detector has a huge dynamic range, its disadvantage is that the number of photodiodes used cannot be reduced easily, and it is less flexible regarding additional outputs. Still this approach might be interesting for future designs.

\section{LIGO}

As in the case of the GEO 600 photodetector, LIGO uses a resonant circuit around the photodiode, as shown in Fig. 4. Components C3 and L3 serve as high-pass filter before amplification of the signal by OP1. The LIGO design uses a relatively high gain of $20 \mathrm{~dB}$ at the amplifier OP1. This has the advantage that an operational amplifier (maximum of 4107 , with minimum stable gain of $20 \mathrm{~dB}$ ) with very low input voltage noise $(0.75 \mathrm{nV} / \sqrt{\mathrm{Hz}})$ can be used, but it has the disadvantage that the maximal possible rf signal size at the detection frequency is comparatively small (i.e., the dynamic range is reduced by this large gain, as discussed in Sec. II A). To overcome problems with limited dynamic range at the detection frequency, LIGO uses an additional feedback loop (called I-servo, not shown) which reduces the part of the signal at the detection frequency that is not suppressed by the interferometer control loop. This is done by adding an appropriate $\mathrm{rf}$ signal, essentially a sine wave at the detection frequency, with the correct amplitude and phase, directly to the photodiode (PD) resonant circuit, thereby canceling the rf signal in one quadrature. The series resonant circuit L4/C4 in the feedback path of OP1 was implemented, to avoid saturation in the amplifier stage at twice the detection frequency.

In contrast to the GEO design, the photocurrent at low frequencies and at dc flows across R10 to ground. The voltage across R10 is amplified by OP2 and serves as a measure of the light power incident on the photodiode. Due to the way the dc photocurrent is detected here, the bias across the photodiode would be decreased by the voltage across R10, and thus depend on the detected light power. To keep the photodiode bias constant instead, the circuit around OP3, OP4, and OP5 is added, which raises the bias at the PD cathode linearly with the voltage across R10. This circuit is not only used to keep the PD bias constant but rather the gain of this feedforward path can be adjusted, such that the phase response of the rf output (at OP1) is as constant as possible 


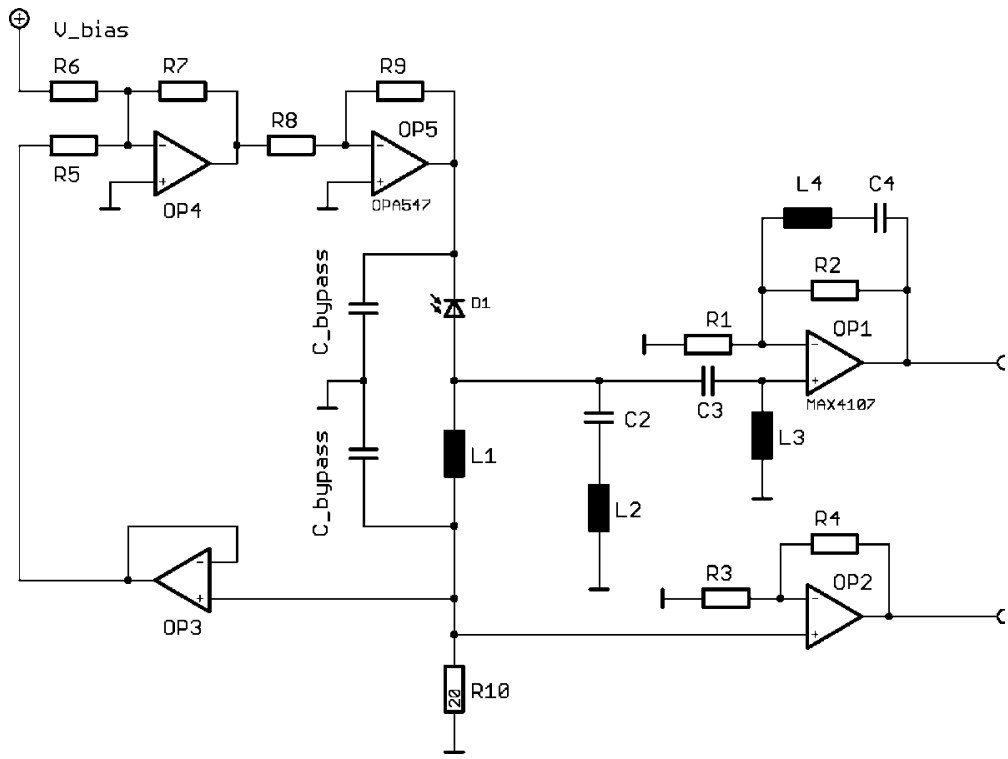

FIG. 4. LIGO photodetector design.

as a function of the detected laser power. This is described in Ref. 6. Naturally, R10 must not be too large, as otherwise the bias voltage needed to compensate for a large signal voltage across R10 would saturate at the output of OP5. Thus with this circuit it is more difficult to reach shot-noise limited sensitivity of the dc path.

\section{VIRGO}

The VIRGO circuit is different, as it processes the photocurrent in an LC network, with the basic idea of using a series resonance circuit consisting of discrete elements $\mathrm{L}$ and $\mathrm{C}$ (L1 and $\mathrm{C} 1$ in Fig. 5), into which the photocurrent flows. The signal voltage is then obtained from the node inside this series resonant circuit, where further passive filtering (e.g., bandpassing and band rejection by $\mathrm{L} 2, \mathrm{C} 2, \mathrm{~L} 3$, and $\mathrm{C} 3$ in Fig. 5) can be applied. As will be discussed in Sec. III, this arrangement has the advantage that the signal voltage does not appear at the PD node in full size. Some explanation of a similar circuit can be found in Ref. 7 .

The dc path is similar to the one in the GEO 600 and TAMA designs, with the difference that the dc output voltage is not generated in the photodiode front end, but on the demodulator board (not shown), at a different physical location. The dc flows via the low-pass filter network L4, C4, and L5

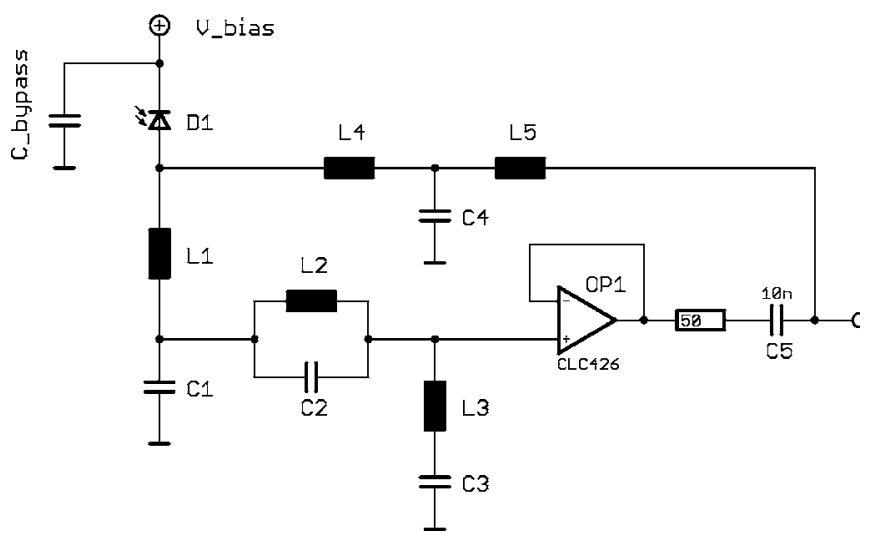

FIG. 5. VIRGO photodetector design. to the output, where on the receiving end (the demodulator board) is a transimpedance stage with very low input impedance at low frequencies. This way the dc photocurrent is flowing on the same physical cable as the rf signal. It remains to be seen if this has major advantages or disadvantages. A possible disadvantage might be that frequency response shaping of the dc signal cannot be done in the front end.

\section{E. TAMA}

The TAMA photodetector is very similar to the GEO 600 design shown in Fig. 3. In addition to the circuitry shown there, TAMA uses a current limiting circuit to prevent the photodiode from damage due to overheating. The design is described in detail in Ref. 8.

\section{THE NEW GEO 600 DESIGN}

Driven by the fact that saturations were observed with large signal levels at the detection frequency with the circuit shown in Fig. 3, a modified circuit was developed that uses a series resonant circuit similar to the VIRGO solution. However, the VIRGO and GEO designs, although similar, were developed independently. A feature of the old design is that the full signal voltage at the detection frequency appears at the PD anode, thereby modulating the bias voltage across the PD.

In the new design, shown in Fig. 6, the photocurrent at the detection frequency flows into the series resonant circuit consisting of $\mathrm{C} 1$ and $\mathrm{L} 1$. The signal then appears as a voltage at the node between $\mathrm{C} 1$ and L1, and is amplified by OP1, currently using a gain of $6 \mathrm{~dB}$.

The essential feature of this circuit is that the impedance "seen" by the PD at the detection frequency is lower than the impedance that is relevant for the current-to-voltage conversion. Ideally the input impedance of the resonant circuit ( $\mathrm{C} 1$ and L1) would be close to $0 \Omega$. However, the capacitance of the PD junction is not negligible and forms a combined reso- 


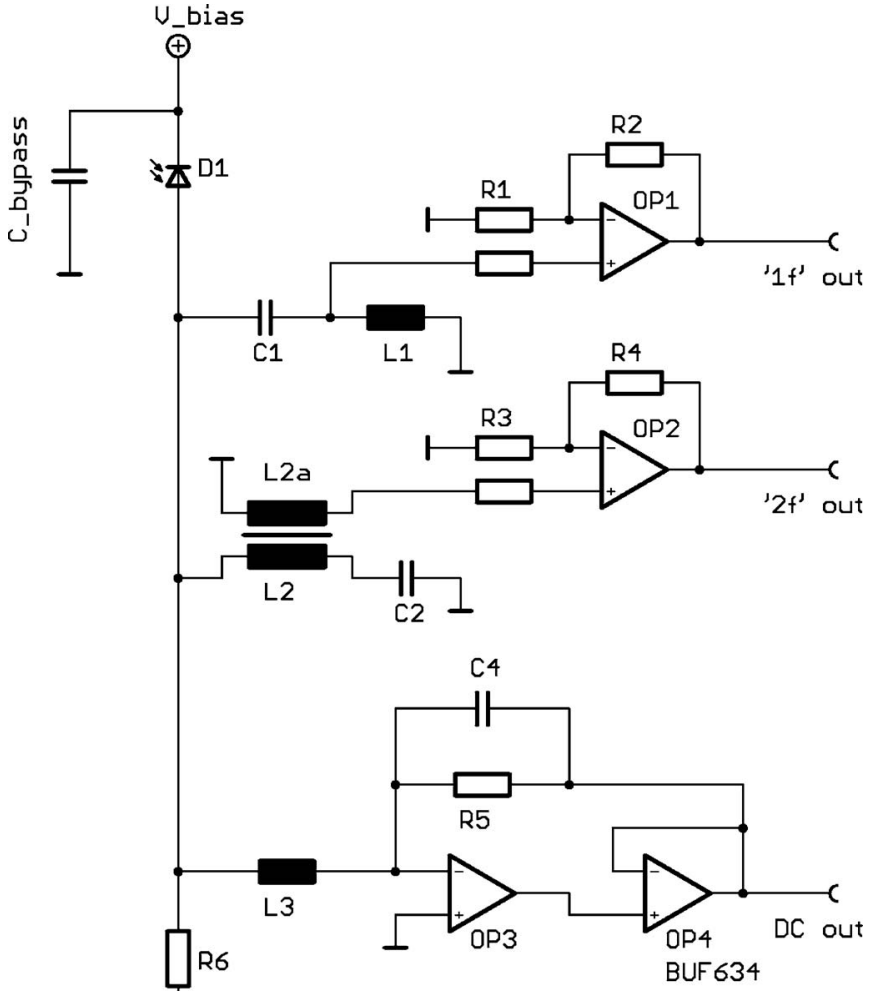

FIG. 6. GEO600 new photodetector design. The resonant circuits L1/C1 and $\mathrm{L} 2 / \mathrm{C} 2$ and the signal extraction around them are just two examples. If the sequence of $\mathrm{L}$ and $\mathrm{C}$ is altered, the shape of the response changes at frequencies different from the resonant frequency. A transformer (as L2/L2a) can be used to provide an optimized signal size.

nant circuit with $\mathrm{C} 1$ and L1. As an example (see Fig. 8) the effective impedance seen by the PD is about $20 \Omega$ while the conversion impedance is about $100 \Omega$.

In addition to the series resonant circuit for the detection frequency, another series resonant circuit was added, consisting of L2 and C2. This works as a notch filter for the double of the detection frequency (called " $2 \mathrm{f}$ "), exactly as is the case for the notch filters in the old GEO 600 and the LIGO design. However, there is also a voltage output added to this notch filter in form of a secondary winding on L2 (L2a) and a voltage amplifier OP2. We plan to use this additional output for future experiments, in which we want the $2 \mathrm{f}$ signal to be detected at the dark port with the maximal light power available. Therefore, it is useful to generate this signal from the same photocurrent that is used for the 1f signal anyway. The implementation of resonant circuits C1, L1 and C2, L2 as examples reflects the way the GEO 600 detector is currently built. However, they just show different possible concepts of signal generation, which can be used simultaneously, to generate a combined multiple resonant photodetector with multiple (optimized) outputs. There can be even more resonant circuits with further outputs if required.

The dc path is still the same as in the old GEO 600 design. An additional frequency response shaping stage with differential sending to twisted pair cables (not shown) is added downstream of OP4. R6 was intended to yield a low impedance for photocurrents at intermediate frequencies (above dc and below the detection frequency). However, R6 must not be too low for noise reasons and possibly can be omitted completely.

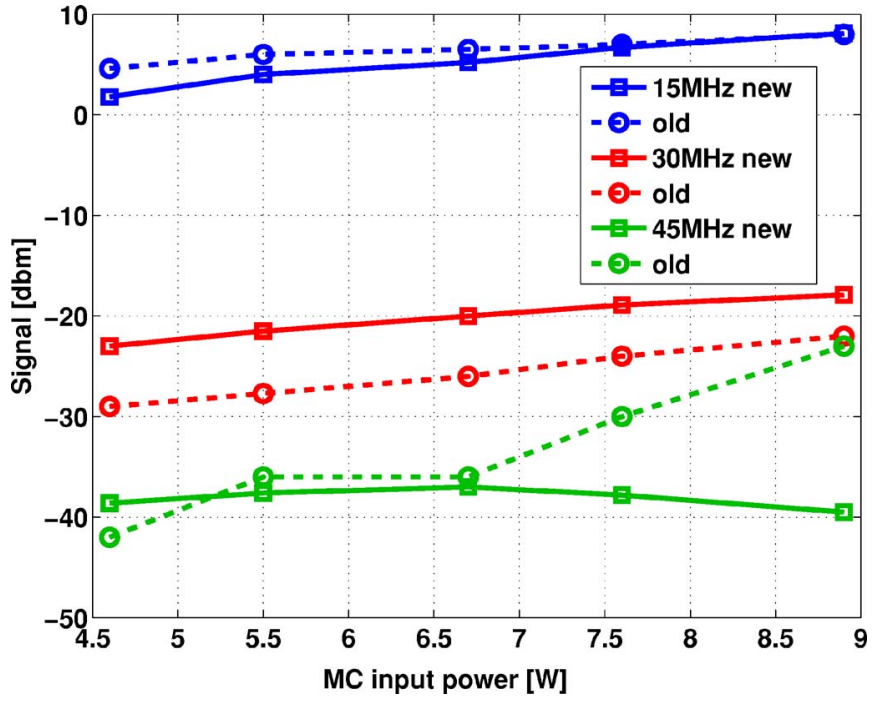

FIG. 7. (Color online) Measurement of signal sizes at three radio frequencies, for the old and new GEO600 photodetector designs. The signal sizes are measured as a function of the laser power.

\section{EXPERIMENTAL RESULTS}

Using the old GEO 600 design, we observed saturation of the rf signal while increasing the light power incident on the photodiode. We suspected that the circuit topology (i.e., the signal voltage generation at the PD anode) might be related to the saturation, and, in fact, the new design did not exhibit this kind of saturation observed with the old design.

Figure 7 shows a measurement of the rf signal size at the output of OP1 for the old (dashed lines) and new (solid lines) GEO 600 PD designs. Both measurements were done under the same conditions with a fully locked interferometer. The voltages were measured with additional buffer operational amplifiers (not shown in Fig. 6), sampling the OP1 output. The three frequencies measured were $15 \mathrm{MHz}$ (the detection frequency), and their second and third harmonics were at 30 and $45 \mathrm{MHz}$, respectively. The graph shows the signal sizes in decibel versus the input light power to the mode cleaners, which increased from 4.6 to $8.9 \mathrm{~W}$ during the experiment. The total photocurrent (at dc) in this experiment was $15 \mathrm{~mA}$ for the low power case ( $4.6 \mathrm{~W}$ to the modecleaners) and $31 \mathrm{~mA}$ for the high power case $(8.9 \mathrm{~W}$ to the mode cleaners). This corresponds to about 22 and $45 \mathrm{~mW}$ of light power incident to the photodiode, respectively.

The measurement shows that with increasing light power the total signal increase at $15 \mathrm{MHz}$ is about 2-3 dB larger for the new circuit design, compared to the old one. Another evident difference is the strong rise of the $45 \mathrm{MHz}$ trace of the old design above $6.7 \mathrm{~W}$ of mode cleaner input power. This rise is strongly overproportional with respect to the power increase.

Both observations indicate a saturation in the case of the old circuit design. In particular, the generation of the third harmonic frequency $(45 \mathrm{MHz})$ is typical for the clipping of a sinusoidal signal. As can be seen for the lowest input power, the conversion factor of the two designs is different by about $2 \mathrm{~dB}$. One may argue that the saturation in the new design is just avoided because of this lower conversion factor, and 


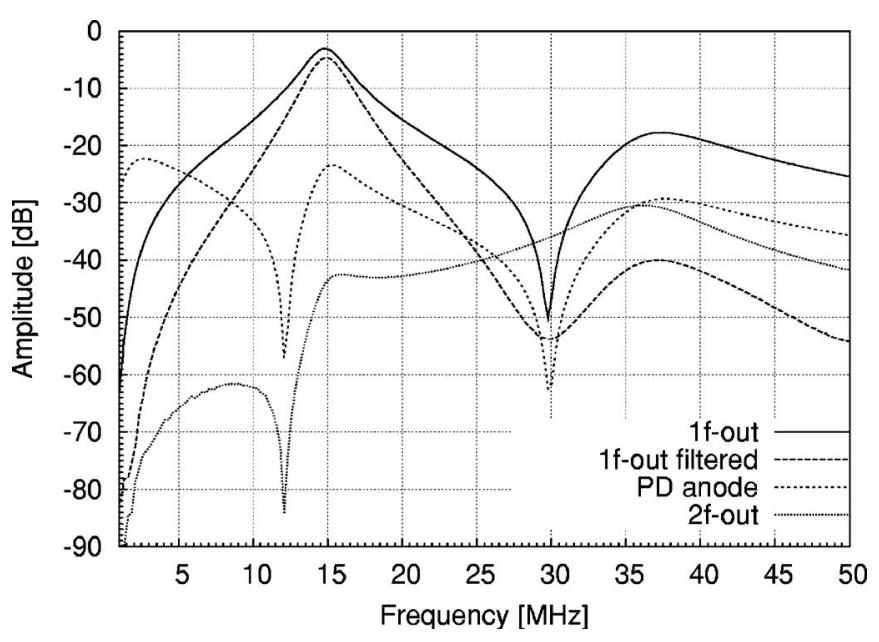

FIG. 8. Measurement of transfer functions from a photodiode test input to different points of the new photodetector circuit.

thus lower maximal signal level. However, it has to be noted that no saturation at this level can be expected from the operational amplifiers used, which are capable of at least $15 \mathrm{dBm}$ output (maximum of 4304 with $+-5 \mathrm{~V}$ power supply). Therefore, the saturation must be located in the passive circuitry before the first operational amplifier.

The noise levels in the vicinity of $15 \mathrm{MHz}$ of the two designs have been observed in this experiment as well. For this purpose the photodiode circuit output is mixed with two (phase-orthogonal) double-balanced frequency mixers, operating with a local oscillator of $15 \mathrm{MHz}$. The output of the mixers is low-pass filtered and amplified, and recorded with a data-acquisition system, sampling with $16 \mathrm{kHz}$. In the region above a few kilohertz of the amplitude spectrum of these signals, the noise was dominated by photon shot noise of the detected light for both designs. The noise levels in the data-acquisition system, combining both quadratures, were $8.1 \mu \mathrm{V} / \sqrt{\mathrm{Hz}}$ for the old and $6.5 \mu \mathrm{V} / \sqrt{\mathrm{Hz}}$ for the new design, respectively.

While the maximum signal level at $15 \mathrm{MHz}$ stayed the same in this experiment, the noise in the case of the new design was lower, thus resulting in a higher signal-to-shotnoise ration of the new design.

For an additional test of distortion and noise, an electrical test signal was applied to the photodiode, such that the output of OP1 had a level of $1 \mathrm{Vrms}$ at the detection frequency. The total harmonic distortion in this case was $-63 \mathrm{dBc}$ and the noise floor was less than $10 \mathrm{nV} / \sqrt{(\mathrm{Hz})}$.

Figure 8 shows measured transfer functions from a test signal input at the PD to various points of the new detector circuit. The trace labeled "1f-out" shows the transfer function to the output of OP1. The circuit is tuned to about $15 \mathrm{MHz}$ (the exact modulation frequency is $14.904915 \mathrm{MHz}$ ). The L2/C2 notch is tuned such that it appears as a notch at about $30 \mathrm{MHz}$ at this output. The trace "1f-out filtered" is the output of OP1, followed by another bandpass for $15 \mathrm{MHz}$, which is normally used before the demodulation of the rf signal. The trace labeled "PD anode" shows the transfer function to the PD anode, and thus gives a measure of the impedance seen by the photodiode. Due to the combination of several series resonant circuits and the effect of the PD capacitance, the resonance (notch) at the PD anode is shifted with respect to the resonance as seen at the output of OP1. However, we have a value of $-24 \mathrm{~dB}$, compared with $-4 \mathrm{~dB}$ in the case of the OP1 output. If we take the gain of OP1 of $6 \mathrm{~dB}$ into account, there remains a difference of $14 \mathrm{~dB}$ in the two transfer functions. This means that the impedance seen by the photodiode at $15 \mathrm{MHz}$ is five times smaller than the impedance of the L1/C1 resonant circuit, which is relevant for the current-to-voltage conversion. Consequently, saturation of this kind observed with the old design should be shifted to a level about five times higher. However, with the current operational amplifier, the detector would be limited at an output level of about $15 \mathrm{dBm}$. Still this is a range increase of roughly a factor of 3 (assuming the saturation starts at about $6 \mathrm{dBm}$ output level in Fig. 7). Finally, the trace labeled " $2 \mathrm{f}$-out" shows the transfer function to the output of OP2 in Fig. 6. The desired resonance is shifted here as well, but there remains some suppression of 1f signal compared with the desired $2 \mathrm{f}$ one.

\section{ACKNOWLEDGMENTS}

The author would like to thank the ILIAS network for support of this work. This document has been assigned LIGO Document No. LIGO-P060032-00-Z.

\footnotetext{
${ }^{1}$ M. B. Gray, D. A. Shaddock, C. C. Harb, and H. A. Bachor, Rev. Sci. Instrum. 69, 3755 (1998).

${ }^{2}$ T. M. Niebauer, R. Schilling, K. Danzmann, A. Rüdiger, and W. Winkler, Phys. Rev. A 43, 5022 (1991).

${ }^{3}$ B. Meers and K. A. Strain, Phys. Rev. A 44, 4693 (1991).

${ }^{4}$ J. B. Johnson, Phys. Rev. 32, 97 (1928).

${ }^{5}$ O. Jennrich, G. Newton, K. D. Skeldon, and J. Hough, Opt. Commun. 205, 405 (2002)

${ }^{6}$ R. Adhikari, (personal communication).

${ }^{7}$ E. Tournefier, (personal communication).

${ }^{8}$ N. Mio, M. Ando, G. Heinzel, and S. Moriwaki, Jpn. J. Appl. Phys., Part 1 40, 426 (2001).
} 